\title{
Lacunar strokes: does shape matter?
}

\author{
Infartos lacunares: o formato da lesão importa?
}

Adriana Bastos Conforto

\author{
Neurologist and Professor, \\ Stroke Group, Clinical Neurology \\ Division, Hospital das Clínicas/ \\ São Paulo University and Center \\ for Interdisciplinary Research on \\ Applied Neurosciences (NAPNA), \\ University of São Paulo. \\ Researcher, Instituto do Cérebro, \\ Hospital Israelita Albert Einstein. \\ São Paulo SP, Brazil. Visiting \\ Professor, Cleveland Clinic, \\ United States of America.

\section{Correspondence} \\ Adriana Bastos Conforto \\ Divisão de Clínica Neurológica, \\ HC/FMUSP \\ Av. Dr. Enéas C. Aguiar 255/5084 \\ 05409-010 São Paulo SP - Brasil. \\ E-mail:adriana.conforto@gmail.com \\ Conflict of interest \\ There is no conflict of interest to \\ declare. \\ Received 12 August 2013 \\ Accepted 20 August 2013
}

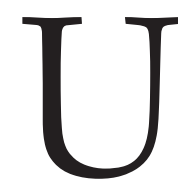

p to one in every four ischemic strokes can be lacunar infarcts. Traditionally, deep brain infarctions with a maximum diameter of $15-20 \mathrm{~mm}$ have been attributed to occlusion of a single penetrating artery by lipohyalinosis or microatheromatosis, but it has been suggested that other etiologies such as embolism may be responsible for up to one third of the cases. Lacunes can be detected by neuroimaging in asymptomatic individuals and are associated with greater risk of cognitive decline $e^{1,2}$.

Lacunar infarcts can have a diameter greater than $15 \mathrm{~mm}$ on axial sections and greater than $20 \mathrm{~mm}$ on coronal or sagittal MRI sections $\mathrm{s}^{3,4}$. It has been argued that shapes of lacunar infarcts may be linked to pathogenesis: lesions with an irregular shape may result from occlusion of largest perforating arteries, or from confluence of lesions, or even from secondary tissue degeneration. Lacunes with ovoid or spheroid, regular shapes may reflect involvement of smallest arteries ${ }^{5}$.

In this issue of Arquivos de Neuro-Psiquiatria, Feng et al. ${ }^{6}$ evaluated clinical and imaging features as well as prognosis of 204 Chinese patients admitted with acute lacunar infarcts, defined as hypointense focal lesions in T1-weighted images and hyperintense focal lesions in T2-weighted, FLAIR, and DWI images with a diameter ranging from $3 \mathrm{~mm}$ to $20 \mathrm{~mm}$. Radiologists (unaware of clinical characteristics) classified infarcts as regular or irregular by visual inspection of MRI images. The authors hypothesized that pathogenesis, clinical symptoms, and prognosis would differ between patients with acute regular or irregular infarcts. Sizes of the infarcts and extent of leukoaraiosis were also evaluated.

Blood pressure, blood glucose, hemoglobin Alc and lipids, as well as blood pressure variability (BPV) were checked within the first 24 hours after stroke. The authors did not mention criteria used to define risk factors for vascular disease such as arterial hypertension, diabetes mellitus, dyslipidemia (history? medical records? measurements/tests performed only before stroke, or either before or after stroke?). Logistic regressions were used to test for associations between risk factors for vascular disease, variation in systolic blood, shape and size of lacunar lesions, extent of leukoaraiosis, neurological deterioration (increase in NIH stroke scale scores at two weeks after stroke), and prognosis (modified Rankin scale at 3 months).

The only variable independently associated with shapes of lacunar infarcts was BPV within the first 24 hours. The authors concluded that "BPV is an independent risk factor for irregularly shaped lacunar infarction". However, because BPV was measured only after stroke, this variable cannot be undoubtedly considered a "risk factor" for irregular infarcts.

Variability in systolic blood pressure has been recognized as a predictor of stroke, and early variability in systolic blood pressure has been associated with worse outcomes after stroke ${ }^{7-9}$. There may be a causal link between BPV and pathogenesis of lacunar infarcts. Still, it is also possible that greater BPV may have occurred only after stroke and, in particular, in more severe strokes. Prospective studies are necessary to clarify this point. Furthermore, locations of infarcts were not depicted, and no information was provided about anti-hypertensive drugs chronically used before stroke, or administered within the first 24 hours after stroke, in patients with regular or irregular infarcts.

The authors also described a significant association between irregular shapes of lacunar infarcts and neurological deterioration two weeks after stroke. More severe leukoaraiosis, infarction size and irregular lacunes were found to be independently associated with worse 
outcomes at 3 months. Since NIH stroke scales within the first 24 hours were not presented, it is uncertain whether or not severities of neurological impairments were comparable at baseline in patients with regular and irregular infarcts. It would be interesting to know whether shapes of the infarcts would remain independently associated with neurological deterioration at 2 weeks or with prognosis at 3 months, if baseline NIH stroke scores had been added to the model.

Efforts have been made to standardize definitions and methods to analyze/report imaging findings in patients with small vessel disease ${ }^{3}$. The work of Feng et al. highlights the importance of searching for distinguished features among patients with "lacunar infarcts". Such lesions should not be underestimated, or considered either "benign" or "homogeneous" strokes ${ }^{2}$. Research addressing the roles of small vessel disease on cognitive decline and neurodegeneration has gained great prominence, in parallel with the escalating increase in life expectancy worldwide, the "epidemics" of vascular disease, and the role played by vascular risk factors on development of small infarcts and leukoaraiosis. Understanding determinants and consequences of different patterns of lacunar infarcts will be valuable to shape preventive, diagnostic and therapeutic measures in order to decrease the burden of small vessel disease worldwide.

\section{References}

1. Bamford J, Sandercock P, Jones L, Warlow P. The natural history of lacunar infarction: the Oxfordshire Community Stroke Project. Stroke 1997;18:545-551.

2. Norrving B. Lacunar infarcts: no black holes in the brain are benign. Pract Neurol 2008;8:222-228.

3. Wardlaw JM, Smith EE, Biessels GJ, et al. Neuroimaging standards for research into small vessel disease and its contribution to ageing and neurodegeneration. Lancet Neurol 2013;12:822-838.

4. Bailey EL, Smith C, Sudlow CLM, Wardlaw JM. Pathology of lacunar ischemic stroke in humans - a systematic review. Brain Pathol 2012;22:583-591.

5. Hervé D. Shape and volume of lacunar infarcts: a 3D MRI study in cerebral autosomal dominant arteriopathy with subcortical infarcts and leukoencephalopathy. Stroke 2005;36:2384-2388.
6. Feng C, Zhang C, Xu Y, Hua T, Liu X, Fang M. Irregularly shaped lacunar infarction: risk factors and clinical significance. Arq Neuropsiquiatr 2013;71:769-773.

7. Rothwell PM. Limitations of the usual blood-pressure hypothesis and importance of variability, instability, and episodic hypertension. Lancet 2010;375:938-948.

8. Rothwell PM, Howard SC, Dolan E, et al. Prognostic significance of visit-to-visit variability, maximum systolic blood pressure, and episodic hypertension. Lancet 2010;375:895-905.

9. Endo K, Kario K, Koga M, et al. Impact of early blood pressure variability on stroke outcomes after thrombolysis: the SAMURAI rt-PA Registry. Stroke 2013;44:816-818.

10. Ryu DW, Shon YM, Kim BS, Cho AH. Conglomerated beads shape of lacunar infarcts on diffusion-weighted MRI: what does it suggest? Neurology 2012;78:1416-1419. 